\title{
Identificação de erros de refração em populares da Região Sul Fluminense
}

\author{
Identification of refractive errors in population of South Fluminense Region \\ Jessica Lagioto Gomes*, William Mikio Ito Otani, Roberta Niquerito Dias, Marina Di Pasqual \\ Granata, Maria da Graça Vaz de Queiroz Silva
}

Resumo

Como citar esse artigo. Gomes JL, Otani WMI, Dias RN, Granata MDG, Silva MGVQ. Identificação de Erros de Refração em Populares da Região Sul Fluminense. Revista de Saúde. 2016 Jan./Jun.; 07 (1): 18-21.

A verificação anual da acuidade visual (AV) em crianças e adultos deve ser recomendada como programa preventivo devido ao alto índice de distúrbios visuais existentes. As últimas estimativas da Organização Mundial da Saúde (OMS) indicam que há 161 milhões de pessoas com alguma deficiência visual em todo o mundo, com um aumento anual de 1 a 2 milhões de indivíduos afetados. Programas de assistência oftalmológica sanitária são necessários como forma de atendimento básico preventivo-educativo, para promover a saúde ocular e encaminhar, precocemente, a população para tratamento adequado. A acuidade visual é o indicador mais imediato da função visual, sendo um exame rápido, simples, de fácil realização e de baixo custo. O método de medida da AV, a partir das tabelas de Snellen e de Jaeger, é preferencialmente usado como triagem em campanhas de saúde visual. A liga de oftalmologia da Universidade Severino Sombra realizou um estudo sobre os erros de refração de populares da região Sul Fluminense, através de feiras de saúde realizadas desde o ano de 2007 até 2011. Participaram desta pesquisa 125 pessoas, sendo o único pré-requisito para esta seleção o fato do indivíduo ter participado em uma das feiras de saúde. O objetivo deste trabalho foi avaliar o déficit visual desta amostra da população, e relacionar a incidência de alterações na AV com o uso correto/incorreto de correção refracional.O uso incorreto é caraterizado através do uso da correção sem necessidade ou com o índice de refração inadequado. Os resultados demonstraram que 32,8\% das pessoas avaliadas tiveram alterações no índice de refração, e $35,2 \%$ faziam uso de correção refracional. Isto indica que $2,4 \%$ da população avaliada utiliza AV inadequadamente. Estes resultados enaltecem a importância do acompanhamento oftalmológico, para diagnóstico da necessidade de correção visual ou suspensão do uso desta sem necessidade.

Palavras-chave: Acuidade visual; Correção refracional; Cegueira.

\begin{abstract}
The annual verification of visual acuity (VA) in children and adults should be recommended as a preventative program, due to the high incidence of visual disturbances. The latest estimates from the World Health Organization (WHO) indicate that there are 161 million visually impaired people worldwide, with an annual increase of 1 to 2 million individuals affected. Eye health and care programs are needed as a form of preventative-educational primary care, to promote eye health and detect early changes and provide appropriate treatment. Visual acuity is the most immediate indicator of visual function, and a quick, simple, easy to perform, and inexpensiveexamination. The AV measuring method, from the Snellen and Jaegertables, is preferably used in screening for eye health during health campaigns. A group of ophthalmology students from Severino Sombra University conducted a study in the population from South Fluminense region, through health fairsfrom 2007 to 2011 . This study surveyed 125 people and only pre requisite was attendance to one of the health fairs. The objective of this study was to evaluate the visual deficit of this population sub-group, and relate the incidence of changes in $\mathrm{AV}$ with the correct or incorrect use ofrefractive correction. Inappropriate use of correction is indicated by use without need or with the wrong refractive index. The results demonstrated that $32.8 \%$ of those evaluated had changes in the refractive index, and $35.2 \%$ were using refractive correction, indicating that $2,4 \%$ of the population studied used inappropriate AV. This highlights the importance of ophthalmological monitoring, diagnosing the need for vision correction or suspension of unnecessary correction.
\end{abstract}

Keywords: Visual acuity; Refractive correction; Blindness.

\section{Introdução}

A verificação anual da acuidade visual (AV) em crianças e adultos é recomendada como atitude preventiva devido ao alto índice de distúrbios visuais existentes. ${ }^{1}$ Estimativas indicam que há 161 milhões de pessoas com deficiência visual em todo o mundo, com um aumento anual de 1 a 2 milhões - isso representa aproximadamente $0,85 \%$ da população mundial. ${ }^{2}$ A prevalência de cegueira aumenta com a idade, principalmente depois dos 50 anos, sendo maior nas áreas rurais e entre as mulheres, provavelmente por causa de sua vulnerabilidade socioeconômica, bem como os obstáculos que enfrentam para acessar os serviços de saúde. ${ }^{3}$

Os problemas visuais acarretam ônus ao aprendizado e à socialização, prejudicando o desenvolvimento natural das aptidões intelectuais, escolares, profissionais e sociais. ${ }^{4}$ Nos países em desenvolvimento encontram-se $80 \%$ dos casos de

Afiliação dos autores: Universidade Severino Sombra, Pró-Reitoria de Ciências Médicas, Curso de Medicina, Vassouras-RJ, Brasil.

*Endereço para correspondência: Universidade Severino Sombra, Av. Exped. Oswaldo de Almeida Ramos, 280 - Centro - Vassouras, RJ - CEP $27700-000$.

E-mail: jessicalgomes@terra.com.br 
cegueira existentes no mundo, sendo dois terços compostos de casos passíveis de prevenção ou curáveis. $^{5}$ Programas de assistência oftalmológica sanitária são necessários como forma de atendimento básico preventivo-educativo, para promover a saúde ocular e encaminhar, precocemente, a população para o tratamento adequado. ${ }^{6}$

A medida da acuidade visual central é o método primordial de avaliação da saúde do sistema visual, sendo o principal auxílio diagnóstico usado pelo médico geral e também o primeiro e principal auxílio diagnóstico do exame oftalmológico especializado. $\mathrm{O}$ instrumento para medida da AV é obrigatório em todos os consultórios oftalmológicos. O resultado do teste de AV é o principal indicativo para avaliar o benefício e desfecho em intervenção oftalmológica cirúrgica e farmacológica, sendo também um dos principais objetivos em pesquisas oftalmológicas..$^{7-8}$

A medida da AV é um bom indicador da saúde visual populacional e de acesso ao serviço especializado de saúde. O acompanhamento do resultado da mesma é útil em saúde pública para prevenir doenças e complicações oftalmológicas como na retinopatia diabética. A medida da visão é fundamental para avaliar tratamentos em protocolos de saúde pública. ${ }^{9}$

A acuidade visual é um indicador mais imediato da função visual, sendo um exame rápido, simples, de fácil realização e de baixo custo. Para as ciências visuais, e particularmente para a oftalmologia, padronizar e quantificar a visão são passos fundamentais para estudar, diagnosticar e tratar as enfermidades oculares. ${ }^{7}$, 10-11 Este estudo teve como objetivo avaliar a prevalência de déficits de acuidade visual, de histórico familiar de doenças oculares e de uso de lentes de correção.

O uso inadequado de correções visuais é caracterizado pelo uso da correção sem a necessidade da mesma, ou através do uso de um índice de correção de refração inadequado para a necessidade do cliente.

\section{Métodos}

Este foi um estudo observacional de desenho transversal e com coleta individual de dados que aconteceu por intermédio da Liga Acadêmica de Oftalmologia do curso de Medicina da Universidade Severino Sombra (USS) de Vassouras, Rio de Janeiro. Este estudo foi aprovado pelo Comitê de Ética em Pesquisa da USS e os participantes assinaram um Termo de Consentimento Livre e Esclarecido de acordo com a Resolução 464 de 2012 do Conselho Nacional de Saúde.

A amostra do estudo foi composta por populares abordados em cinco oportunidades ao longo dos anos 2007, 2008, 2009, 2010 e 2011 em feiras de saúde realizadas na cidade de Vassouras. O único critério para a avaliação do indivíduo foi a expressão da vontade de ter sua acuidade visual avaliada.

As tabelas de Snellen e Jaeger foram usadas como triagem de defeitos da acuidade visual. Os participantes foram separados em três grupos: 1) queixa oftalmológica atual; 2) história oftalmológica pregressa; 3) história familiar de distúrbio oftalmológico. Foram também coletados dados sobre a profissão de cada participante, além de suas etnicidade.

O percentual de casos de uso incorreto de correção visual foi calculado subtraindo-se o percentual de clientes que utilizavam correção do percentual de clientes que efetivamente demonstraram alterações no índice de refração.

Este é um estudo puramente descritivo, com resultados reportados em unidades de prevalência por meio de gráficos. Os dados foram anotados em formulários de registro e posteriormente digitados em banco de dados no software Microsoft Excel.

\section{Resultados}

Participaram deste estudo 125 indivíduos. A Figura 1 descreve a divisão dos indivíduos nos três grupos previamente citados, divididos por etnicidade, indicando uma prevalência de indivíduos de etnia branca. Houve uma prevalência de queixas atuais e de histórico familiar entre os participantes. A Figura 2 ilustra a distribuição dos indivíduos nos três grupos de acordo com atividade profissional.

Após o exame foi evidenciado que $32,8 \%$ das pessoas avaliadas tiveram alterações no índice de refração, e 35,2\% faziam uso de correção refracional; demonstrando que $2,4 \%$ faziam uso da mesma sem necessidade. Este cálculo subestima o uso de correção inadequado, visto que não são computados os clientes que utilizam correção e possuem alteração no índice de refração, mas cuja correção não equivale à suas necessidades. Os detalhes da avaliação de acuidade visual podem ser encontrados na Figura 3.

\section{Discussão}

Os dados coletados por este estudo trazem a inferência de que o interesse e adesão às consultas oftalmológicas pela população sul fluminense é baixo já que apesar dos consideráveis índices de problemas oculares muitos indivíduos ainda se encontram em tratamento. A falta de acesso à correção refracional está entre a lista das maiores causas de cegueira no país, seguida de doenças como a catarata, o glaucoma, o diabetes, além de degeneração macular associada à idade. $^{12}$

De acordo com o Conselho Brasileiro de Oftalmologia, existem atualmente no Brasil um milhão 


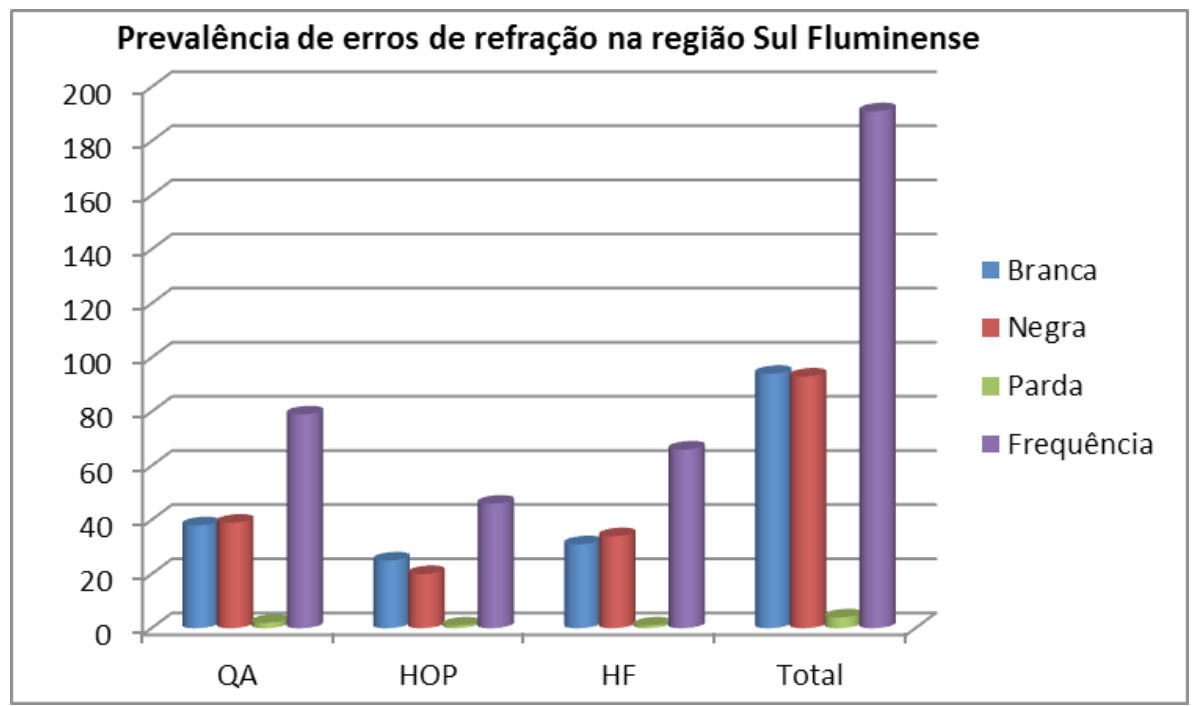

Figura 1. Prevalência de erros de refração de acordo com etnia, e divisão de acordo com a presença de queixa oftalmológica atual,históriaoftalmológica pregressa ou história oftalmológica familiar

$\mathrm{QA}=$ queixa atual; $\mathrm{HOP}=$ história oftalmológica pregressa; $\mathrm{HF}=$ história familiar.

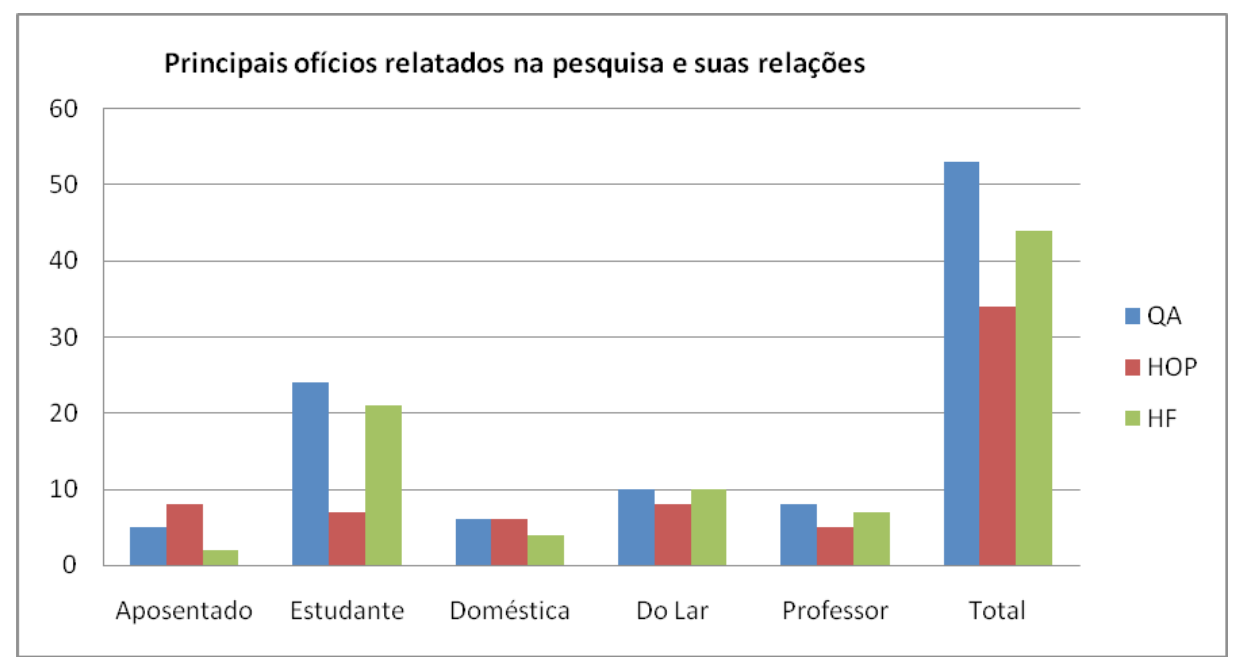

Figura 2. Relação dos ofícios relatados e distribuição dos grupos por ofício $\mathrm{QA}=$ queixa atual; $\mathrm{HOP}=$ história oftalmológica pregressa; $\mathrm{HF}=$ história familiar.

\section{Resultados}
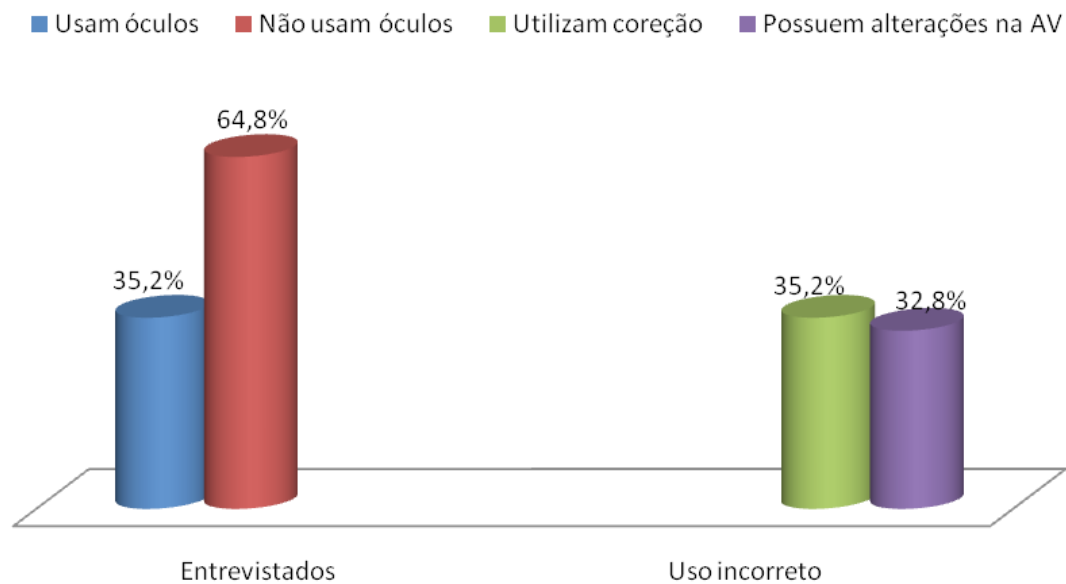

Figura 3. Resultado final do estudo de campo, relacionando os entrevistados e o uso correto e incorreto de correção refracional. 
de cegos e quatro milhões de deficientes visuais. ${ }^{6}$ Muitas doenças oculares são detectadas por meio de exames periódicos, assim como o glaucoma, considerado a segunda maior causa de cegueira irreversível no mundo cujas consequências podem ser evitadas através do diagnóstico precoce. ${ }^{13}$

Existem doenças graves e prevalentes que podem ser diagnosticadas precocemente por um oftalmologista através de exames oculares periódicos. A catarata, principal causa de cegueira tratável no mundo, é uma delas. A ambliopia, que se não for tratada antes dos sete ou oito anos de idade pode acarretar em déficit visual importante, e o ceratocone (alteração da córnea que provoca um afinamento progressivo de sua porção central e consequente baixa da acuidade visual) também podem ser facilmente diagnosticados. ${ }^{14}$

A prevalência de exame de acuidade visual alterado encontrada por este estudo pode ser considerada alta quando comparada com o estudo de Neto etal., que avaliou a acuidade visual de crianças em idade escolar e obteve uma prevalência de um pouco mais de $7 \%$ de exames alterados..$^{15}$ Vale ressaltar que este estudo pode contar com um viés de seleção já que necessitava da voluntariedade de seus participantes e há uma tendência de que os doentes busquem atenção à saúde em relação aos indivíduos previamente hígidos.

\section{Considerações finais}

Conclui-se que houve ocorrência importante de alterações ao exame de acuidade visual, apesar da maioria da população estudada não usar correção quando a mesma está indicada. Tal fato ressalta a importância de eventos que avaliem a acuidade visual de indivíduos e a eventual necessidade de acompanhamento oftalmológico.Estas medidas tornam-se importantes na prevenção de doenças que podem causar cegueira e demais complicações.

\section{Declarações}

Os autores não possuem conflitos de interesse diretos ou indiretos. A fonte de financiamento deste estudo não foi divulgada.

\section{Referências}

1. Ministério da Saúde do Brasil. Projeto Olhar Brasil: triagem de acuidade visual: manual de orientação. Brasília, 2008.

2. Benjamin W J. Borish's Clinical Refraction. Second Edition. St. Louis: ButterworthHeinemann; 2006.

3. Resnikoff S, Pascolini D, Etya'ale D, Kocur I, Pararajasegaram R, Pokharel GP, MariottI SP. Global data on visual impairment in the year 2002. Bull WHO. 2004;82(11):844-51.

4. Gianini RJ, Mais E, Coelho EC, Oréfice FR, Moraes RA. Prevalência de baixa acuidade visual em escolares da rede pública, Sorocaba. Rev. Saúde Pública 2004: 38(2):201-8.

5. Taleb A, Faria MAR, Ávila M, Mello PAM. As condições de saúde ocular no Brasil - 2012. Conselho Brasileiro de Oftalmologia. São Paulo, 2012.

6. Conselho Brasileiro de Oftalmologia. Mais acesso a saúde ocular. Edição ampliada e revisada. São Paulo, 2015.

7. Kronbauer AL,Schor P, Carvalho LAV. Medida da visão e testes psicofísicos. Arq. Bras. Oftalmol2008;71(1):122-7.

8. Sugano MD, Giorgi MM, Serra DC, Souza PR, Rehder JRCL. Resultados obtidos com a medida da acuidade visual em alunos de uma escola de nível primário da rede privada no $\mathrm{ABC}$ paulista. Revista brasileira de oftalmologia 2005;64(3):183-90.

9. FILHO A,et al. Prevalence of visual impairment, blindness, ocular disorders and cataract surgery outcomes in low-income elderly from a metropolitan region of São Paulo - Brazil. Arq. Bras. Oftalmol., São Paulo, v. 71 , n. 2, Apr. 2008

10. Campbell FW, Green DG. Optical and retinal factors affecting visual resolution. J Physiol. 1965;181(3):576-93.

11. Bicas HEA. Acuidade visual: medidas e notações. Arq Bras Oftalmol. 2002;65(3):375-84.

12. Bicas HE, Matsushima EH, Silva JA. Visão e percepção visual. Arq Bras Oftalmol. 2003;66(1):5-8.

13. Brito PR, Veitzman S. Causas de cegueira e baixa visão em crianças. Arq Bras Oftalmol. 2000;63(1):49-54.

14. Kara-José N, Almeida GV, Arieta CEL, Araújo JS, Becgara SJ, Oliveira PR. Causas de deficiência visual em crianças. Bol Sanit Panam. 1994;97(5): 405-12.

15. Neto M, Augusto C, Ramos AT, Bugmann L. Relação entre acuidade visual e condições de trabalho escolar em crianças de um colégio do ensino fundamental público de Curitiba. Rev. bras.oftalmol 2014; 73(4): 216-19. 